\title{
On Projective Representations of Finite Wreath Products
}

\author{
By John R. Durbin and K. Bolling Farmer
}

\begin{abstract}
The theory of induced projective representations is applied to finite wreath products, yielding algorithms which add to the collection of groups for which projective representations can be computed systematically. For finite Abelian and Abelianwreath-cyclic groups, the factor sets are determined explicitly by establishing a oneto-one correspondence between certain lower triangular matrices and the inequivalent factor sets of these two classes of groups. This correspondence is used to determine the number and degrees of the inequivalent, irreducible projective representations.
\end{abstract}

1. Introduction. The problem of determining a complete set of inequivalent irreducible linear (ordinary) representations of a finite group is difficult unless the group is extremely uncomplicated; this has been discussed in a number of works, including the paper by Dixon [5], for example. The case of projective representations is all the more difficult. In this paper we add to the list of groups for which projective representations can be computed systematically by applying the theory of induced projective representations to finite wreath products. The corresponding application for linear representations has been considered by several authors; see, in particular, Kerber [9], [10] and Durbin [6].

In Section 2 we describe in detail an algorithm for constructing explicitly the projective representations of wreath products, including several remarks concerning computational efficiency. In Section 3 the factor sets of Abelian and Abelian-wreathcyclic groups are derived in terms of matrices. Expressing these maps as arrays is a crucial step in programming the algorithm. This correspondence is further used to obtain information about the number and degrees of the irreducible projective representations. In Section 4, we illustrate the procedure for the specific group $Z_{3} \sim Z_{3}$.

Groups are assumed finite and representations are over the field of complex numbers. We discuss only standard wreath products; this reduces notational difficulties but still brings out the essential ideas. The symbol $\sim$ denotes equivalence of representations. In addition to the various specific references we mention the general references [4], [8] and [11].

Let $A$ and $B$ denote groups, and $K=A^{B}$ the set of functions from $B$ to $A$, made into a group by componentwise multiplication. For $k \in K$ and $b \in B$, define $k^{b} \in K$ by $k^{b}(y)=k\left(y b^{-1}\right)$ for all $y \in B$. Then, for each $b \in B$, the mapping $k \mapsto k^{b}$ is an automorphism of $K$, and the extension of $K$ by the group of all such

Received February 23, 1976.

AMS (MOS) subject classifications (1970). Primary 20C25, 20F25; Secondary 68A10.

Key words and phrases. Wreath products, Abelian groups, projective representations, factor sets, induced representations, algorithm. 
automorphisms is the wreath product of $A$ and $B, A \sim B$. We identify $K$ and $B$ with the obvious subgroups of $A \sim B$. Then $K \triangleleft A \sim B, K \cap B=\{e\}$, and $K B=A \sim B$; typical elements will be denoted by $k b$.

2. Construction of Projection Representations of $A \sim B$. We describe an algorithm for constructing a complete set of inequivalent, irreducible projective representations of $A \sim B$, based on the method of induced representations, following Mackey [12] and Backhouse [1].

I. We first express a factor set of $A \sim B$ in terms of the following theorem:

THEOREM 1. Every factor set $\omega^{\prime}$ of $A \sim B$ is equivalent to a factor set $\omega$ of $A \sim B$ which may be uniquely represented in the form

$$
\omega\left(k_{1} b_{1}, k_{2} b_{2}\right)=\gamma\left(k_{1}, b_{1} k_{2} b_{1}^{-1}\right) \beta\left(b_{1}, k_{2}\right) \alpha\left(b_{1}, b_{2}\right),
$$

where $\gamma$ is a factor set of $K, \alpha$ is a factor set of $B$, and $\beta: B \times K \rightarrow T$ (the circle group) satisfies:

$$
\beta\left(b, k_{1} k_{2}\right)=\beta\left(b, k_{1}\right) \beta\left(b, k_{2}\right) \gamma\left(b k_{1} b^{-1}, b k_{2} b^{-1}\right) / \gamma\left(k_{1}, k_{2}\right)
$$

and

$$
\beta\left(b_{1} b_{2}, k\right)=\beta\left(b_{1}, b_{2} k b_{2}^{-1}\right) \beta\left(b_{2}, k\right) .
$$

Moreover, for every choice of $\gamma, \alpha$, and $\beta$ satisfying (i) and (ii), the function $\omega$ defined by (*) is a factor set of $A \sim B$.

The results in Section 3 are useful in generating a complete set of inequivalent factor sets of $A \sim B$ decomposed according to Theorem 1 .

II. The next step is to construct a complete set of inequivalent irreducible $\omega$ representations of $A \sim B$ for the factor set $\omega$ decomposed in terms of the factor sets $\gamma$ of $K, \alpha$ of $B$, and the function $\beta$.

Let $D$ be an irreducible $\gamma$-representation of $K$ of degree $d$. We associate with $D$ two subgroups of $A \sim B$ : the inertia group $H_{D}=\left\{x \in A \sim B: D^{x} \sim D\right\}$, where for all $k \in K$

$$
D^{x}(k)=\left(\omega\left(x^{-1}, k x\right) \omega(k, x) / \omega\left(x^{-1}, x\right)\right) \cdot D\left(x^{-1} k x\right),
$$

and the inertia factor $H_{D}^{*}=\left\{b \in B: D^{b} \sim D\right\}$. We note that the $x$-conjugate $D^{x}$ of $D$ is defined so that it has the same factor set $\gamma$ as $D$. It is easily shown that $H_{D}$ is the semidirect product of $K$ and $H_{D}^{*}$. To extend $D$ to a representation of $H_{D}$, we use the fact that for each $b \in H_{D}^{*}$ there exists a nonsingular matrix, say $T_{b}$, such that $D^{b}(k)=T_{b}^{-1} D(k) T_{b}$ for all $k \in K$. The irreducibility of $D$ implies that the map $b \longmapsto T_{b}$ is a $\lambda$-representation of $H_{D}^{*}$ for some factor set $\lambda$ of $H_{D}^{*}$. To find the equivalence operators $T_{b}$, it is sufficient to consider only generators of $K$ and $B$, thus yielding a smaller set of equations. Furthermore, Backhouse [1] gives a formula for computing $T_{b}$. To determine the factor set $\lambda$ of $T$, we need only compare a corresponding entry in the matrices $T_{b_{1} b_{2}}$ and $T_{b_{1}} T_{b_{2}}$ for all $b_{1}, b_{2}$ in $H_{D}^{*}$.

Let $\left\{V_{j}: 1 \leqslant j \leqslant n\right\}$ be a complete set of inequivalent, irreducible $\alpha \lambda$-representations of $H_{D}^{*}$. The set $\left\{U_{j}: 1 \leqslant j \leqslant n\right\}$, where $U_{j}: H_{D} \rightarrow \mathrm{GL}(d, C)$ is defined by 


$$
U_{j}(k b)=D(k) T_{b} \otimes V_{j}(b)
$$

is a set of $\omega$-representations of $H_{D}$. By induction we lift this set to a set of irreducible $\omega$-representations $\left\{U_{j} \uparrow A \sim B\right\}$ of $A \sim B$. Again, care must be taken to preserve factor sets; see [2].

To obtain a complete set of inequivalent $\omega$-representations of $A \sim B$ we must consider orbits of the $\gamma$-representations of $K$. The orbit of the irreducible $\gamma$-representation $D$ of $K$ is the set

$$
\begin{array}{r}
\mathcal{O}_{D}=\left\{D^{\prime}: D^{\prime} \text { is an irreducible } \gamma \text {-representation of } K\right. \\
\text { and } \left.D^{\prime} \sim D^{b} \text { for some } b \in B\right\} .
\end{array}
$$

Then a complete set of inequivalent, irreducible $\gamma$-representations of $K$ is the union of disjoint orbits, say $O_{D_{1}} \cup \cdots \cup O_{D_{m}}$. By carrying out the method given above for each $D_{i}, 1 \leqslant i \leqslant m$, we obtain

$$
\left\{U_{i j} \uparrow A \sim B: 1 \leqslant i \leqslant m, 1 \leqslant j \leqslant n_{i}\right\},
$$

a complete set of inequivalent, irreducible $\omega$-representations of $A \sim B$.

As a final remark, this step begins with a $\gamma$-representation of $K$. To construct such a representation, the algorithm outlined in I and II is applicable. We note that for a direct sum the steps simplify considerably.

III. We obtain all irreducible inequivalent projective representations of $A \sim B$ by repeating I and II for each factor set in a complete set of inequivalent factor sets of $A \sim B$.

3. Abelian-Wreath-Cyclic Groups. We now apply the preceding algorithm to obtain specific information about the irreducible projective representations of finite Abelian and Abelian-wreath-cyclic groups. We prove that the factor sets of these two classes of groups can be explicitly described in terms of matrices. This expression enables us to determine the number and degrees of the inequivalent, irreducible projective representations.

Let $A$ be finite Abelian, say $A=Z_{n_{1}} \oplus \cdots \oplus Z_{n_{s}}$ with $n_{i} \mid n_{i+1}$ for $1 \leqslant i \leqslant$ $s-1$, and $B$ finite of order $t$. We first consider the base group of $A \sim B$. If $\left\langle a_{i}\right\rangle=$ $Z_{n_{i}}$, then $\left\{a_{i}: 1 \leqslant i \leqslant s\right\}$ generates $A$ and $K$ has a set of generators $\left\{x_{1}, \ldots, x_{s t}\right\}$, where $x_{l}$ corresponds to $a_{i}$ for $i \equiv l(\bmod s)$. Thus each element $x$ of $K$ can be expressed as

$$
x=x_{1}^{u_{1}} x_{2}^{u_{2}} \cdots \cdots x_{s t}^{u_{s t}}
$$

$0 \leqslant u_{l} \leqslant n_{i}-1$, where $i \equiv l(\bmod s)$. We identify $x$ with the vector $\left(u_{1}, \ldots, u_{s t}\right)^{T}$. We have the following theorem about the factor sets of $K$.

THEOREM 2. Let $G$ be the direct sum of $m$ cyclic groups, say $G=G_{1} \oplus \cdots$ $\oplus G_{m}$, with corresponding generators $x_{i}$ of order $n_{i}$ such that $n_{i} \mid n_{i+1}, 1 \leqslant i \leqslant m-1$. The elements of $G$ are identified with $m$-tuples as described above. Let $\gamma^{\prime}$ be a factor set of $G$. Then $\gamma^{\prime}$ is equivalent to a factor set $\gamma$ with a corresponding $m \times m$ lower triangular matrix $G_{\gamma}$ such that

$$
\gamma(x, y)=\exp \left(-2 \pi i x^{T} G_{\gamma} y\right) .
$$


The $i$, jth component $g_{i, j}$ of $G_{\gamma}$, for $i>j$, is an element of the set

$$
C_{i, j}=\left\{l / N_{i, j}: l \in Z, 0 \leqslant l \leqslant N_{i, j}-1, N_{i, j}=\left(n_{i}, n_{j}\right)\right\} .
$$

Furthermore, if the values of the $i$, $j$ th entries range independently over $C_{i, j}$ for $1 \leqslant j$ $<i \leqslant m$, then the $G_{\gamma}$ 's correspond to a complete set of inequivalent factor sets of $G$.

Operations involving these matrices are done modulo 1 . In order to prove this theorem and several others, we need the following fact proved by Backhouse.

Lemm A (BACKноUSE [1, p. 278]). Let $\omega$ be a factor set of an Abelian group $G$. Then $\omega$ is equivalent to the trivial factor set if and only if $\omega$ is symmetric, that is, $\omega(x, y)=\omega(y, x)$ for all $x$ and $y$ in $G$.

Now let us prove Theorem 2 .

Proof. Let $x, y$, and $z$ be elements of $G$. Let $G_{\gamma}$ be an $m \times m$ lower triangular matrix with its $i$, jth entry from $C_{i, j}(1 \leqslant j<i \leqslant m)$. If $\gamma: G \times G \rightarrow C$ is defined by

$$
\gamma(x, y)=\exp \left(-2 \pi i x^{T} G_{\gamma} y\right)
$$

then it follows that $\gamma$ is a factor set of $G$.

We now must show that for two factor sets $\gamma$ and $\delta$ with corresponding matrices $G_{\gamma}$ and $G_{\delta}$, if $G_{\gamma} \neq G_{\delta}$, then $\gamma$ is not equivalent to $\delta$. If $\gamma \sim \delta$, then the function $(x, y) \mapsto \gamma(x, y) / \delta(x, y)$ is equivalent to the trivial factor set of $G$. Thus, $G_{\gamma}-G_{\delta}$ must be a symmetric matrix and so $G_{\gamma}=G_{\delta}$.

Since the number $\Pi\left|C_{i, j}\right|$ of distinct matrices is $\Pi\left(n_{i}, n_{j}\right)$ for $1 \leqslant j<i \leqslant m$, which $\check{Z}_{\text {mud }}[13$, p. 5] proves to be the order of $M(G)$, we conclude that this is a complete set of inequivalent factor sets.

Two corollaries follow.

Corollary 1. Let $\gamma$ be a factor set of an Abelian group $G$ such that for each $x$ and $y$ in $G$,

$$
\gamma(x, y)=\exp \left(-2 \pi i x^{T} G_{\gamma} y\right)
$$

for some $m \times m$ matrix $G_{\gamma}$. Then $\gamma$ is equivalent to the trivial factor set if and only if $G_{\boldsymbol{\gamma}}$ is a symmetric matrix.

Corollary 2. Let $A=Z_{n_{1}} \oplus Z_{n_{2}} \oplus \cdots \oplus Z_{n_{s}}$ with $n_{i} \mid n_{i+1}$ for $1 \leqslant i<$ $s-1$, and let $B$ have order $t$. If $G_{\gamma}$ is an $s t \times$ st lower triangular matrix whose $i$, jth entry $g_{i, j} \in C_{i, j}$, where

$$
\begin{aligned}
C_{i, j}=\left\{l / N_{i, j}: l \in Z, 0 \leqslant l \leqslant N_{i, j}-1, N_{i, j}=\left(n_{i_{0}}, n_{j_{0}}\right),\right. \\
\left.i_{0} \equiv i \bmod s, j_{0} \equiv j \bmod s\right\},
\end{aligned}
$$

$(1 \leqslant j<i \leqslant s t)$, then $\gamma: K \times K \rightarrow C$, defined for all $x$ and $y$ in $K$ by

$$
\gamma(x, y)=\exp \left(-2 \pi i x^{T} G_{\gamma} y\right)
$$

is a factor set of $K$. If the values of the $i$, jth entries range independently over $C_{i, j}$ for $1 \leqslant j<i \leqslant s t$, then the $G_{\gamma}$ 's correspond to a complete set of inequivalent factor sets of $K$.

Using Frucht's result [7], we are able to specify the number and degrees of the inequivalent; irreducible $\gamma$-representations of the base group for a given factor set $\gamma$ in terms of the matrix $G_{\boldsymbol{\gamma}}$. 
THEOREM 3. Let $G$ be the direct sum of $m$ cyclic groups $G_{1} \oplus \cdots \oplus G_{m}$ such that $G_{i}$ is generated by $x_{i}$ of order $n_{i}$ and $n_{i} \mid n_{i+1}, 1 \leqslant i \leqslant m-1$. Let $\gamma$ be a factor set of $G$ with corresponding matrix $G_{\gamma}$. The number of inequivalent, irreducible $\gamma$ representations of $G$ is the number $n_{\gamma}$ of solutions of the form $\left(u_{1}, \ldots, u_{m}\right)$ to the following set of $m$ congruences:

$$
\left\{\begin{array}{rr}
u_{2} g_{2,1}+u_{3} g_{3,1}+u_{4} g_{4,1}+\cdots+u_{m} g_{m, 1} \equiv 0 & \bmod 1 \\
-u_{1} g_{2,1}+u_{3} g_{3,2}+u_{4} g_{4,2}+\cdots+u_{m} g_{m, 2} \equiv 0 & \bmod 1 \\
-u_{1} g_{3,1}-u_{2} g_{3,2}+u_{4} g_{4,3}+\cdots+u_{m} g_{m, 3} \equiv 0 & \bmod 1 \\
\cdot & \\
\cdot & \\
-u_{1} g_{m, 1}-u_{2} g_{m, 2}-\cdots-u_{m-1} g_{m, m-1} \equiv 0 \bmod 1
\end{array}\right.
$$

Proof. We note that the matrix of coefficients appearing in this set (*) is $G_{\gamma}-G_{\gamma}^{T}$. Frucht [7] proved that the number of inequivalent, irreducible $\gamma$-representations of $G$ is the order of the subgroup

$$
\{x \mid \gamma(x, y)=\gamma(y, x) \text { for all } y \text { in } G\} .
$$

Thus, we want to find necessary and sufficient conditions for $x=x_{1}^{u_{1}} \cdots \cdots x_{m}^{u_{m}}$ to be in this subgroup.

Let $y=x_{1}^{v_{1}} \cdots \cdots x_{m}^{v_{n}}$ be an element of $G$. Then

$$
\begin{aligned}
\gamma(x, y)=\exp [- & 2 \pi i\left(u_{2} g_{2,1}+u_{3} g_{3,1}+\cdots+u_{m} g_{m, 1}\right) v_{1} \\
& \left.+\left(u_{3} g_{3,2}+\cdots+u_{m} g_{m, 2}\right) v_{2}+\cdots+\left(u_{m} g_{m, m-1}\right) v_{m-1}\right]
\end{aligned}
$$

and

$$
\begin{aligned}
\gamma(y, x)=\exp [- & 2 \pi i\left(v_{2} g_{2,1}+\cdots+v_{m} g_{m, 1}\right) u_{1} \\
& \left.+\left(v_{3} g_{3,2}+\cdots+v_{m} g_{m, 2}\right) u_{2}+\cdots+\left(v_{m} g_{m, m-1}\right) u_{m-1}\right] .
\end{aligned}
$$

If $\gamma$ is symmetric with respect to $x$, then $\gamma\left(x, x_{i}\right) \doteq \gamma\left(x_{i}, x\right)$ for $1 \leqslant i \leqslant m$. This fact implies the following equations:

$$
\begin{aligned}
& \exp \left[-2 \pi i\left(u_{2} g_{2,1}+u_{3} g_{3,1}+\cdots+u_{m} g_{m, 1}\right)\right]=\exp [-2 \pi i \cdot 0]=1, \\
& \exp \left[-2 \pi i\left(u_{3} g_{3,2}+\cdots+u_{m} g_{m, 2}\right)\right]=\exp \left[-2 \pi i\left(g_{2,1} u_{1}\right)\right], \\
& \quad \cdot \\
& \quad \cdot \\
& \exp \left[-2 \pi i\left(u_{j+1} g_{j+1, j}+u_{j+2} g_{j+2, j}+\cdots+u_{m} g_{m, j}\right)\right] \\
& =\exp \left[-2 \pi i\left(g_{j, 1} u_{1}+g_{j, 2} u_{2}+\cdots+g_{j, j-1} u_{j-1}\right)\right] \\
& \quad \cdot \\
& \quad \cdot \\
& 1=\exp \left[-2 \pi i\left(g_{m, 1} u_{1}+g_{m, 2} u_{2}+\cdots+g_{m, m-1} u_{m-1}\right)\right] .
\end{aligned}
$$


So we have the set $(*)$ of $m$ congruences as a necessary condition.

Furthermore, if $x=x_{1}^{u_{1}} \ldots x_{m}^{u_{m}}$ and $\left(u_{1}, \ldots, u_{m}\right)$ satisfies $(*)$, then $\gamma(x, y)=\gamma(y, x)$ for all $y$ in $G$; that is, this condition is sufficient.

Therefore, for $A$ Abelian, a complete set of factor sets of the base group $K$ of $A \sim B$ can be described explicitly. In addition, the number and degree of the inequivalent, irreducible $\boldsymbol{\gamma}$-representations of $K$ for a given $\gamma$ can be calculated in terms of this description.

The next question we must answer is this: For a given factor set $\gamma$ of $K$, when does there exist a function $\beta: B \times K \rightarrow C$ so that $\left(k_{1} b_{1}, k_{2} b_{2}\right) \mapsto \gamma\left(k_{1}, b_{1} k_{2} b_{1}^{-1}\right) \cdot$ $\beta\left(b_{1}, k_{2}\right)$ is a factor set of $A \sim B$ ? We call such a factor set $\gamma$ compatible. The following theorem gives the answer for $A$ Abelian and $B$ cyclic.

THEOREM 4. Let $A$ be finite Abelian with torsion decomposition $Z_{n_{1}} \oplus \cdots$ $\oplus Z_{n_{s}}$ and $B$ cyclic of order $t$. Let $\gamma$ be a factor set of $K$ with corresponding matrix $G_{\gamma} . A$ necessary and sufficient condition for $\gamma$ to be compatible is that $C G_{\gamma} C^{T}-G_{\gamma}$ be symmetric, where $C$ is the st $\times$ st matrix

$$
C=\left[\begin{array}{cccccc}
0 & 0 & \cdot & \cdots & 0 & 1 \\
1 & 0 & \cdot & \cdots & 0 & 0 \\
0 & 1 & 0 & \cdots & 0 & 0 \\
\cdot & & & & & \\
\cdot & & & & & \\
0 & 0 & & \cdots & 1 & 0
\end{array}\right]^{s} .
$$

Proof. Let $b$ be a generator of $B$. We note that $C$ is the matrix representing the action of $b$ on $K$.

By Mackey's decomposition, $\gamma$ is compatible if and only if there exists a function $\beta: B \times K \rightarrow T$ such that

(i) $\beta\left(b^{n}, k_{1} k_{2}\right)=\beta\left(b^{n}, k_{1}\right) \beta\left(b^{n}, k_{2}\right) \gamma\left(b^{n} k_{1} b^{-n}, b^{n} k_{2} b^{-n}\right) / \gamma\left(k_{1}, k_{2}\right)$,

(ii) $\beta\left(b^{n} b^{m}, k\right)=\beta\left(b^{n}, b^{m} k b^{-m}\right) \beta\left(b^{m}, k\right)$, and

(iii) $\beta\left(e_{B}, k\right)=\beta\left(b^{n}, e_{K}\right)=1$,

for all $k_{1}, k_{2}$, and $k$ in $K$ and for $0 \leqslant n, m \leqslant t-1$.

We first prove that the condition is necessary. Suppose that $\gamma$ is compatible.

From (i), we see that the factor set $\theta$ of $K$ defined by

$$
\theta\left(k_{1}, k_{2}\right)=\gamma\left(b k_{1} b^{-1}, b k_{2} b^{-1}\right) / \gamma\left(k_{1}, k_{2}\right)
$$

must be symmetric. The matrix corresponding to $\theta$ is $C G_{\gamma} C^{T}-G_{\gamma}$; thus, by Corollary 1 of Theorem 3, we have that $\theta$ is symmetric if and only if $C G_{\gamma} C^{T}-G_{\gamma}$ is symmetric. Hence the symmetry of $C G_{\gamma} C^{T}-G_{\gamma}$ is certainly a necessary condition for the compatibility of $\gamma$.

To prove that the symmetry of $C G_{\gamma} C^{T}-G_{\gamma}$ is sufficient, we construct a function $\beta$ which satisfies (i), (ii), and (iii). We define $\beta: B \times K \rightarrow T$ by

(1) $\beta(b, k)=\exp \left[-\pi i k^{T}\left(C G_{\gamma} C^{T}-G_{\gamma}\right) k\right]$, and

(2) $\beta\left(b^{n}, k\right)=\Pi_{l=0}^{n-1} \beta\left(b, b^{l} k b^{-l}\right)$.

It follows that if $C G_{\gamma} C^{T}-G_{\gamma}$ is symmetric and if we define $\beta$ in this manner, then $\boldsymbol{\gamma}$ is compatible. 
In order to state the resulting corollary, we must introduce notation concerning the matrices $\left\{G_{\gamma}: \gamma \in M(K)\right\}$ for $A, B, \gamma$, and $G_{\gamma}$ as given in Theorem 4. Consider the $s t-1$ subdiagonals below the main diagonal of $G_{\gamma}$. The subdiagonal whose first component is $g_{s t-m+1,1}$ is referred to as the complement of the subdiagonal whose first component is $g_{m+1,1}$; that is, the complement of the $m$ th subdiagonal is the $(s t-m)$ th subdiagonal. We see the $m$ th subdiagonal has $s t-m$ entries and that its complementary $(s t-m)$ th subdiagonal has $m$ entries for $1 \leqslant m \leqslant s t / 2$ if $s t$ is even and $1 \leqslant m \leqslant$ $(s t-1) / 2$ if $s t$ is odd. If $s t$ is even, then the $s t / 2$ th subdiagonal is its own complement.

Corollary. The compatible factor sets of the base group $K$ of $A \sim B$ can be described in terms of their corresponding matrices (see Corollary 2 of Theorem 1).

(1) Assume s and $t$ are odd. The corresponding matrix $G_{\gamma}$ of a compatible factor set $\gamma$ of $K$ has the following form: each of the first $(s t-1) / 2$ subdiagonals is determined by a cycle of s elements. The complementary subdiagonals are obtained by taking the negatives of the continuation and repetition of the s-cycle. That is, the mth subdiagonal consists of repetitions of the s-cycle $\left\{g_{m+1,1}, g_{m+2,2}, \ldots, g_{m+s, s}\right\}$. If the last entry in the mth subdiagonal is $g_{m+i, i}$, then the complementary subdiagonal begins with $-g_{m+i+1, i+1}, \ldots,-g_{m+s, s}$ and continues with negatives of the s-cycle.

(2) Assume $s$ is even and $t$ is odd. For a compatible factor set $\gamma$ of $K$, the first st/2 - 1 subdiagonals and their complements are constructed as in (1). The st/2th subdiagonal has as its s-cycle $\left\{g_{l+1,1}, g_{l+2,2}, \ldots, g_{l+s / 2, s / 2},-g_{l+1,1}, \ldots,-g_{l+s / 2, s / 2}\right\}$, where $l=s t / 2$. Thus its s-cycle is determined by $s / 2$ elements.

(3) Assume $t$ is even. The first st/2-1 subdiagonals and their complements in the matrix $G_{\gamma}$ for a compatible $\gamma$ are formed as in (1). The st/2th subdiagonal has as its s-cycle $\left\{g_{l+1,1}, g_{l+2,2}, \ldots, g_{l+s, s}\right\}$, where $l=s t / 2$ and $g_{l+i, k} \in C_{l+i, i}$ such that $g_{l+i, i}=-g_{l+i, i}$.

As an example, consider $\left(Z_{4} \oplus Z_{8}\right) \sim Z_{4}$. Its base group has $4^{22} 8^{6}$ inequivalent factor sets, $4^{6} 8$ being compatible. The compatibles have corresponding matrices:

$$
\left[\begin{array}{rrrrrrrr}
0 & 0 & 0 & 0 & 0 & 0 & 0 & 0 \\
a_{1} & 0 & 0 & 0 & 0 & 0 & 0 & 0 \\
b_{1} & a_{2} & 0 & 0 & 0 & 0 & 0 & 0 \\
c_{1} & b_{2} & a_{1} & 0 & 0 & 0 & 0 & 0 \\
d_{1} & c_{2} & b_{1} & a_{2} & 0 & 0 & 0 & 0 \\
-c_{2} & d_{2} & c_{1} & b_{2} & a_{1} & 0 & 0 & 0 \\
-b_{1} & -c_{1} & d_{1} & c_{2} & b_{1} & a_{2} & 0 & 0 \\
-a_{2} & -b_{2} & -c_{2} & d_{2} & c_{1} & b_{2} & a_{1} & 0
\end{array}\right]
$$

where $a_{l}, b_{l}$, and $c_{l}$ belong to their respective $C_{i, j}(1 \leqslant l \leqslant 2), d_{1} \in\{0,2 / 4\}$, and $d_{2} \in\{0,4 / 8\}$.

As we have seen, the compatible factor sets of the base group can be explicitly determined. If $\gamma$ is such a factor set, then $\gamma$ may be extended to more than one factor set of $A \sim B$; that is, there may be more than one function satisfying (i), (ii), and 
(iii) in the proof of Theorem 4 corresponding to $\gamma$. However, in the next theorem, we see that in certain cases, each compatible factor set need only be extended once to obtain a complete set of inequivalent factor sets of $A \sim B$.

Theorem 5. Let $A=Z_{n_{1}} \oplus \cdots \oplus Z_{n_{s}}$ such that $n_{i} \mid n_{i+1}, 1 \leqslant i \leqslant s-1$, and let $B=Z_{t}$. Let $c$ denote the number of compatible factor sets of the base group of $A \sim B$. If $t$ is odd or if $n_{1}$ and $t$ are even, then $c=|M(A \sim B)|$.

Proof. Using Blackburn's theorem [3] to calculate $|M(A \sim B)|$, we obtain:

for $t$ odd,

$$
|M(A \sim B)|=\left[n_{1}^{(s+3(s-1))} n_{2}^{(s-1+3(s-2))} \cdots \cdots \cdot n_{s-1}^{(2+3)} n_{s}\right]^{(t-1) / 2},
$$

and for $t$ even,

$$
|M(A \sim B)|=2^{s}\left[n_{1}^{(s+3(s-1))} n_{2}^{(s-1+3(s-2))} \cdots \cdots n_{s-1}^{(2+3)} n_{s}\right]^{(t / 2-1)} .
$$

We determine $c$ by straightforward computation of the number of values which can appear as components in a compatible matrix. When $s$ and $t$ are odd, $c=\Pi\left|C_{i, j}\right|$ for $i>j, 2 \leqslant i \leqslant(s t+1) / 2$, and $1 \leqslant j \leqslant s$. If $s$ is even and $t$ odd,

$$
c=\Pi\left|C_{i, j}\right| \cdot \Pi\left|C_{m+1, l}\right|,
$$

and if $n_{1}$ and $t$ are even,

$$
\grave{c}=\Pi\left|C_{i, j}\right| \cdot 2^{s},
$$

where $i>j, m=s t / 2,2 \leqslant i \leqslant m$, and $1 \leqslant l \leqslant s / 2$. In each case, $c=|M(A \sim B)|$.

4. Example. We apply these results to find the irreducible projective representations of $Z_{3} \sim Z_{3}$.

The base group $K$ of $Z_{3} \sim Z_{3}$ has 3 compatible factor sets $\gamma_{1}, \gamma_{2}$ and $\gamma_{3} ; \gamma_{2}$, for example, corresponds to

$$
\left[\begin{array}{ccc}
0 & 0 & 0 \\
1 / 3 & 0 & 0 \\
2 / 3 & 1 / 3 & 0
\end{array}\right]
$$

Using Theorem 3, we see that $K$ has exactly 27 inequivalent, irreducible (linear) $\gamma_{1}$ representations of degree 1,3 inequivalent, irreducible $\gamma_{2}$-representations of degree 3 , and 3 such $\gamma_{3}$-representations of degree 3 . We construct these by applying the algorithm given in Section 2.

The factor sets of $K$ are extended to $Z_{3} \sim Z_{3}$ via functions constructed in the proof of Theorem 4; let these new factor sets be $\omega_{1}, \omega_{2}$, and $\omega_{3}$. By determining orbits and inertia factors, we find that a complete set of inequivalent, irreducible representations of $Z_{3} \sim Z_{3}$ consists of 17 linear $\omega_{1}$-representations (9 of degree 1 and 8 of degree 3), $1 \omega_{2}$-representation of degree 9 , and $1 \omega_{3}$-representation of degree 9 . Furthermore, we can describe these representations explicitly. An irreducible $\omega_{2}$ representation is $T \uparrow Z_{3} \sim Z_{3}$, where $T$ is an $\gamma_{2}$-representation of $K$ of the form 


$$
T\left(x_{1}{ }_{1} x_{2}{ }^{u_{2}} x_{3}^{u_{3}}\right)=a^{u_{3}}\left[\begin{array}{lll}
1 & 0 & 0 \\
0 & a & 0 \\
0 & 0 & a^{2}
\end{array}\right]^{u_{1}}\left[\begin{array}{lll}
0 & 0 & 1 \\
1 & 0 & 0 \\
0 & 1 & 0
\end{array}\right]^{u_{2}}\left[\begin{array}{ccc}
0 & 1 & 0 \\
0 & 0 & a^{-1} \\
a^{-2} & 0 & 0
\end{array}\right]^{u_{3}}
$$

for $a=\exp (2 \pi / 3)$.

Department of Mathematics

The University of Texas

Austin, Texas 78712

Department of Mathematics

University of Florida

Gainesville, Florida 32601

1. N. B. BACKHOUSE, "Projective representations of space groups. II, Factor systems," Quart. J. Math. Oxford Ser. (2), v. 21, 1970, pp. 277-295. MR 43 \#7517.

2. N. B. BACKHOUSE, "Projective representations of space groups. III, Symmorphic space groups," Quart. J. Math. Oxford Ser. (2), v. 22, 1971, pp. 277-290. MR 45 \#2028.

3. N. BLACKBURN, "Some homology groups of wreathe products," Illinois J. Math., v. 16, 1972, pp. 116-129. MR 45 \#388.

4. C.W. CURTIS \& I. REINER, Representation Theory of Finite Groups and Associative Algebras, Interscience, New York and London, 1962. MR 26 \#2519.

5. J. D. DIXON, "Computing irreducible representations of groups," Math. Comp., v. 24, 1970, pp. 707-712. MR 43 \#6330.

6. J. R. DURBIN, “On locally compact wreath products," Pacific J. Math., v. 57, 1975, pp. 99-107. MR 51 \#13125.

7. R. FRUCHT, "Über die Darstellung endlicher abelscher Gruppen durch Kollineationen," J. Reine Angew. Math., v. 166, 1931, pp. 16-29.

8. B. HUPPERT, Endliche Gruppen. I, Springer-Verlag, Berlin and New York, 1967. MR 37 \#302.

9. A. KERBER, “Zur Darstellungstheorie von Kranzprodukten," Canad. J. Math., v. 20, 1968, pp. 665-672. MR 38 \#2223.

10. A. KERBER, Representations of Permutation Groups. I, Lecture Notes in Math., vol. 240, Springer-Verlag, Berlin and New York, 1971. MR 48 \#4098.

11. L. JANSEN \& M. BOON, Theory of Finite Groups. Applications in Physics, NorthHolland, Amsterdam; Interscience, New York, 1967. MR 36 \#6490.

12. G. W. MACKEY, “Unitary representations of group extensions. I," Acta Math., v. 99, 1958, pp. 265-311. MR 20 \#489.

13. È. M. ŽMUD', "Symplectic geometric and projective representations of finite abelian groups," Mat. Sb. (N.S.), v. 87 (129), 1972, pp. 3-17 = Math. USSR Sb., v. 16, 1972, pp. 1-16. MR $45 \# 2044$. 